\title{
UNE ANALYSE DES DOCUMENTS ÉTRANGERS PUBLIÉS DANS LA PRESSE PÉRIODIQUE DU SOCIALISME ARGENTIN (1894-1905)
}

\author{
Lucas Poy, traduit de l'espagnol par Lucie Guesnier
}

Société d'études jaurésiennes | « Cahiers Jaurès »

$2019 / 4 \mathrm{~N}^{\circ} 234$ | pages 35 à 56

ISSN 1268-5399

Article disponible en ligne à l'adresse :

https://www.cairn.info/revue-cahiers-jaures-2019-4-page-35.htm

Distribution électronique Cairn.info pour Société d'études jaurésiennes.

(C) Société d'études jaurésiennes. Tous droits réservés pour tous pays.

La reproduction ou représentation de cet article, notamment par photocopie, n'est autorisée que dans les limites des conditions générales d'utilisation du site ou, le cas échéant, des conditions générales de la licence souscrite par votre établissement. Toute autre reproduction ou représentation, en tout ou partie, sous quelque forme et de quelque manière que ce soit, est interdite sauf accord préalable et écrit de l'éditeur, en dehors des cas prévus par la législation en vigueur en France. Il est précisé que son stockage dans une base de données est également interdit. 


\title{
Une analyse des documents étrangers publiés dans la presse périodique du socialisme argentin (1894-1905)
}

\author{
Lucas Poy
}

Dans le dernier tiers du XIX $\mathrm{X}^{\mathrm{e}}$ siècle et dans la première décennie $\mathrm{du} \mathrm{XX}^{\mathrm{e}}$ siècle, la fusion entre le mouvement ouvrier et les idées socialistes semblait devenir réalité dans une grande partie de l'Europe occidentale mais aussi, de manière embryonnaire, dans d'autres parties du monde. Les années d'activité de la Deuxième Internationale (18891914) furent caractérisées par un climat d'optimisme général, ouvrant de nombreuses perspectives pour un ensemble de partis socialistes qui agrandirent ainsi leur influence et développèrent leur capacité organisationnelle. Ce fut une période de croissance pour la classe ouvrière qui s'organisait en syndicats et qui voyait augmenter sans discontinuer les effectifs des nouveaux partis ouvriers de masse acquis au socialisme, et s'accroître leurs résultats électoraux ${ }^{1}$.

1 Sur l'histoire de la Deuxième Internationale voir, entre autres, Patricia Van Der Esch, La Deuxième Internationale 1889-1923, Paris, Marcel Rivière, 1957 ; Giuliano Procacci, "Studi sulla Seconda Internazionale e sulla Socialdemocrazia tedesca ", Annali del Istituto Giangiacomo Feltrinelli, 1, 1958, pp. 105-146 ; Georges Haupt, La Deuxième Internationale, 1889-1914 : étude critique des sources, essai bibliographique, Paris, Mouton, 1964 ; George Douglas Howard Cole, A history of socialist thought, vol. 3, part 1/2, The second international, 1889-1914, London, Macmillan, 1963 ; James Joll, The Second International, 1889-1914, New York, Harper \& Row, 1966 ; Julius Braunthal, Geschichte der Internationale, Berlin, Dietz, 1978 ; Franco Andreucci, "La difusión y la vulgarización del marxismo ", in Eric Hobsbawm (dir.) Historia del marxismo, Barcelona, Bruguera, 1980 ; Jacques Droz (dir.), Histoire générale du socialisme, de 1875 à 1918, vol. 2, Paris, PUF, 1997 ; Igor Krigovuz, The Second International 1889-1914, Moscou, Progress Publishers, 1989 et Gary Steenson, After Marx, before Lenin : Marxism and socialist working-class parties in Europe, 1884-1914, Pittsburgh, University of Pittsburgh Press, 1991. 
Peu avant le grand cataclysme du milieu de l'année 1914, Lénine, dans l'hommage qu'il écrivit à l'occasion de la mort du dirigeant socialiste allemand August Bebel, observait rétrospectivement l'histoire et notait qu'après une longue période marquée, d'une part, par la " naissance des idées socialistes » et de l'autre, par « les premières manifestations de la lutte du prolétariat ", le dernier tiers du XIX ${ }^{\mathrm{e}}$ siècle avait finalement été la période "de formation, de développement et de maturation des partis socialistes de masse composés de la classe prolétarienne ». Cette étape se caractérisait, de son point de vue, par la " propagation considérable du socialisme, l'augmentation inhabituelle de toutes sortes d'organisations de prolétaires et la préparation complète du prolétariat dans les domaines les plus divers, pour l'accomplissement de sa grande mission historique ${ }^{2}$ ».

Quelques années auparavant, dans Que faire?, le leader bolchevik avait annoncé que «ceux qui [connaissaient] tant soit peu la situation de fait de notre mouvement ne [pouvaient] pas ne pas voir que la large diffusion du marxisme [avait] été accompagnée d'un certain abaissement du niveau théorique ${ }^{3}$ ». Cette remarque faisait référence à ce qu'on appelait « la diffusion et la vulgarisation du marxisme ", une question importante déjà traitée dans l'historiographie. Franco Andreucci évoquait la nécessité de problématiser le processus qui avait conduit à la fusion entre le marxisme - élaboration théorique et politique spécifique, née sous la plume de Karl Marx et Friedrich Engels, à un moment précis du XIX ${ }^{\mathrm{e}}$ siècle - et le mouvement ouvrier, dont le développement avait fait un bond décisif partout dans le monde au cours du dernier tiers du même siècle. Selon l'auteur, cette période avait été marquée par un processus « d'expansion et d'appauvrissement, de propagation et de schématisation " car « les voies de son affirmation étaient aussi celles de sa systématisation, et les mécanismes de sa diffusion avaient fini par appauvrir son patrimoine originel ». "C'était l'une de ces rares occasions, résumait l'auteur italien, où un ensemble d'idées (...) avaient fusionnées avec un grand mouvement social ascendant exprimant une forte demande idéologique ${ }^{4}$. " Dans un fameux

2 Vladimir Ilitch LÉnine, "August Bebel ", in Lenin Collected Works, Moscou, Progress Publishers, vol. 19, 1977 [1913], pp. 295-301.

3 V. I. LÉNINe, Que faire ?, 1902, (édition en ligne : http://www.centremlm.be/ IMG/pdf/que_faire.pdf), p. 8.

4 Franco Andreucci, "La difusión y la vulgarización del marxismo », art. cit., p. 15 , p. 28 
article traitant de sujets similaires, Eric Hobsbawm remarquait que « le marxisme lui-même était un produit des années 1890. Le terme n'était apparu, du moins dans les titres des articles de journaux, que vers le milieu de cette décennie ${ }^{5}$ ».

Il s'agit donc ici de comprendre quand eut lieu cette rencontre, mais aussi de quel type de marxisme il s'agissait. Dans quelle mesure le mouvement ouvrier de la fin du XIX ${ }^{\mathrm{e}}$ siècle et du début du siècle suivant s'appropria-t-il cette idéologie, sachant qu'elle se manifestait de manières variables dans différentes parties du monde? Nous identifions communément le «marxisme " à un certain corpus théorique et politique, mais il convient d'en historiciser le contenu afin d'en saisir les particularités dans le contexte historique et national que nous analysons ici. Enfin, comme nous le verrons dans cet article, dans les textes que les socialistes diffusaient au cours de cette période, ils ne se référaient pas seulement au marxisme - même compris dans son sens le plus large - mais s'appuyaient également sur une myriade de courants théoriques alternatifs, tels que le positivisme, le darwinisme social et d'autres idées influentes de la philosophie contemporaine, ce qui complexifie encore davantage l'analyse.

Quels sont les textes qui circulaient à cette époque parmi les militants du socialisme argentin et quels en étaient les auteurs? Quelles furent les modalités concrètes de diffusion du socialisme en Argentine, et comment s'articula sa "vulgarisation » simultanément aux premières expériences organisationnelles et politiques du mouvement ouvrier? Cet article vise à apporter une contribution à la reconstruction historique nécessaire des chemins qu'emprunta le mouvement ouvrier en Argentine à la fin du XIX siècle et au début du XX $\mathrm{XX}^{\mathrm{e}}$ siècle lorsqu'il entra en contact avec les idées socialistes. Le choix de la coupure chronologique se base sur l'importance de la décennie 1895-1905, située à cheval sur les deux siècles. D’une part cette décennie laissa des traces profondes dans la structuration politique et idéologique du socialisme dans ce pays. De l'autre, elle correspond à la chronologie de La Vanguardia, l'organe de presse officiel du parti socialiste argentin sur lequel s'appuie cette recherche, depuis son apparition en avril 1894 jusqu'à sa transformation en quotidien à la fin de l'année 1905.

5 Eric Hobsваwм, "La difusión del marxismo (1890-1905)", in Marxismo e historia social, Puebla, Instituto de Ciencias de la Universidad Autónoma de Puebla, 1983, p. 102. 
Pendant cette décennie, le journal paraissait de manière hebdomadaire - à l'exception d'une brève période pendant laquelle il parut deux fois par semaine - et structurait l'activité du Parti socialiste autant d'un point de vue idéologique qu'organisationnel. L'analyse exhaustive de près de six cents numéros de La Vanguardia parus au cours de cette décennie nous a permis de dresser une liste de tous les articles publiés à partir de textes étrangers. Des chroniques de congrès internationaux aux textes sur l'histoire de la philosophie, en passant par des extraits d'ouvrages classiques de Marx et des petites publications de matériel de propagande produites par les dirigeants et militants du socialisme européen, cet ensemble de titres les plus divers représente un vaste corpus, mis à disposition à d'éventuelles autres recherches et qui constitue le support fondamental de cette analyse. Dans son étude sur l'accueil de Marx en Argentine, Horacio Tarcus identifia « quatre moments dans le processus de diffusion du marxisme en Argentine »: les années 1870, pendant lesquelles les émigrants français jouèrent un rôle fondamental ; les années 1880, pendant lesquelles les émigrants allemands les remplacèrent ; les années 1890, pendant lesquelles le marxisme fut expliqué "dans les termes du socialisme scientifique " et adossé à la Deuxième Internationale ; et enfin une période qui se situe entre 1895 et 1910, caractérisée par « l'intégration de la pensée de Marx au domaine naissant des sciences sociales ${ }^{6} »$. Pour cette recherche, nous nous plaçons dans la coupe chronologique située entre le troisième et le quatrième moment de Tarcus, bien que nous nous intéressions davantage aux contributions étrangères (marxistes ou non) qui paraissaient dans la presse périodique du Parti socialiste qu’à la réception des idées de Marx.

Il ne fait aucun doute que l'établissement de listes d'articles et de brochures publiées ne suffit pas à mesurer l'ensemble des influences intellectuelles et politiques sur le socialisme argentin. Néanmoins, nous considérons que cette reconstruction est une étape nécessaire permettant d'avoir une idée plus précise - clarifiant un ensemble d'impressions et d'intuitions générales - de ce que les socialistes argentins lisaient et diffusaient au tournant du siècle. De quels pays et de quels périodiques venait la majorité des articles publiés ? Quels furent les auteurs les plus retranscrits ? Quelles brochures se vendaient à l'époque? Où étaient-elles éditées? Voici quelques-

6 Horacio TARcus, Marx en la Argentina. Sus primeros lectores obreros, intelectuales y cientificos, Buenos Aires, Siglo XXI, 2007, p. 46. 
unes des questions auxquelles il s'agit de répondre dans cet article, en se basant sur les données recueillies et analysées pour cette recherche.

\section{La Vanguardia, étude de cas}

Au milieu des années 1890, plusieurs centaines de militants adhéraient au socialisme argentin. Ils étaient répartis dans divers groupes et clubs locaux qui entraient progressivement dans un processus de centralisation. Au cours de la décennie suivante, le socialisme avait agrandi son influence, les organisations étaient plus stables et l'opinion publique s'intéressait davantage à l'idéologie ${ }^{7}$. Ses militants développèrent une rhétorique politique dans les usines et les ateliers, sur la voie publique, dans les assemblées et les réunions ouvrières. Ils consacrèrent sans relâche les quelques heures qui leur restaient après de longues journées de travail à la propagande et au travail d'organisation. Il s'agissait à la fois de stimuler leurs collègues et en même temps, d'élaborer une stratégie politique claire destinée à changer le système social et économique, considéré comme la cause de toutes les exploitations.

La sensibilisation orale n'était pas la seule manière de mener le combat. En effet, dès les premières années, la presse écrite joua un rôle de premier plan dans le développement de la propagande au sein des milieux ouvriers. Les brochures, les livres et surtout les journaux - imprimés dans le pays ou importés de l'étranger grâce aux liens très actifs

7 La littérature sur le socialisme argentin est très vaste et en pleine expansion. Voici quelques exemples de travaux généraux sur le PS au cours de cette période initiale : Jacinto Oddone, Historia del socialismo argentino, Buenos Aires, La Vanguardia, 1934 ; Richard Walter, The Socialist Party of Argentina, 1890-1930, Austin, The University of Texas Press, 1977 ; Jeremy Adelman, "Socialism and Democracy in Argentina in the Age of the Second International ", The Hispanic American Historical Review, 72 (2), 1992 ; Hernán Camarero, Carlos Herrera, «El Partido Socialista en Argentina : nudos históricos y perspectivas historiográficas ", in Hernán Camarero, Carlos Herrera (ed.), El Partido Socialista en Argentina, Buenos Aires, Prometeo, 2005 ; Osvaldo Graciano, "El Partido Socialista de Argentina : su trayectoria histórica y sus desafíos políticos en las primeras décadas del siglo XX ", A contra corriente, vol. 7 (3), 2010, pp. 1-37 ; Ricardo Falcón, "Orígenes del movimiento socialista en Argentina. Prólogo. Capítulo I y II ", Cuadernos del Ciesal, n 10, 2011, pp. 11-45; Lucas Poy, "Between the Strike and the Ballot Box : The Early Years of the Argentine Socialist Party, 1890-1910", Journal of Labor and Society, vol. 21, 2018, pp. 37-53. 
que les militants entretenaient avec le mouvement ouvrier européen de l'époque - étaient des éléments essentiels de la lutte, lui donnaient une forme tangible et participaient à la conscientisation des travailleurs. De plus, il n'était pas rare que les imprimés soient utilisés comme support à la propagation orale, soit parce qu'ils fournissaient des éléments de réflexion dans les interventions et les débats publics, soit parce qu'ils étaient directement lus à haute voix durant les réunions auxquelles participaient des travailleurs illettrés.

La diffusion de brochures et de livres occupa un rôle important dans l'organisation des premiers noyaux socialistes urbains. En effet, au début, les militants socialistes se rassemblaient dans de petits groupes qui s'occupaient de faire de la propagande. L'augmentation des interventions dans les rangs des travailleurs, vers la fin des années 1880 et au début de la décennie suivante, ne fit qu'accentuer la nécessité de répandre des textes susceptibles d'expliquer les projets politiques socialistes et de les développer. La propagande était considérée comme une activité essentielle des organisations socialistes : l'élaboration progressive d'un raisonnement pédagogique sur les causes de l'exploitation et les moyens pour les combattre était la condition nécessaire à la mise en place d'actions conjointes et solidaires des travailleurs, à l'abandon de tout conformisme et de toute perspective individuelle. Pour les socialistes argentins, cette culture de l'écrit joua plus généralement un rôle éducatif essentiel et contribua indubitablement à la lutte en faveur de ce qu'ils appelaient « l'élévation morale du prolétariat».

L'émergence des premiers journaux socialistes hispanophones au début des années 1890, mais surtout le lancement de La Vanguardia en avril 1894, fournit au socialisme argentin naissant un outil puissant de propagande. En effet, le journal fonctionnait tellement bien en tant que dispositif d'organisation qu'il précéda de deux ans la constitution officielle du parti, fondé au milieu de l'année 1896. Les pages de l'hebdomadaire associaient des articles éditoriaux sur la situation politique du pays, de nombreuses notes de propagande, ainsi que des chroniques sur les activités du mouvement ouvrier et des annonces de programmes. Le journal fonctionna comme un instrument d'organisation collective, proposant aussi bien des analyses théoriques et politiques qu'un programme d'actions de lutte à mener au quotidien ${ }^{8}$.

8 Sur les caractéristiques de La Vanguardia et sur son évolution jusqu’à sa trans- 
Les articles de doctrine et de propagande étaient principalement écrits par des auteurs étrangers. Il s'agissait d'ouvrages aux caractéristiques très diverses : extraits de livres et de brochures publiés et édités à l'étranger, articles en provenance d'autres journaux socialistes, extraits de romans et chapitres d'ouvrages scientifiques. Dans certains cas, la source à partir de laquelle le matériel avait été prélevé était indiquée ; dans de nombreux autres cas, en revanche, ces informations manquent. Quoiqu'il en soit, dans l'ensemble, ces articles constituent un corpus de sources très précieuses pour comprendre quelles étaient à l'époque les lectures des militants socialistes et de leurs sympathisants.

\section{Évolution quantitative}

La préparation de cet article a nécessité une analyse approfondie de tous les numéros publiés par La Vanguardia entre avril 1894 et fin août 1905, date à laquelle il fut transformé en quotidien. Dans ce corpus de 586 numéros, il a été possible d'identifier un total de 311 articles initialement publiés à l'étranger'. Comme on peut le constater en analysant cette liste, il s'agit de textes hétérogènes tant du point de vue de longueur, que du style et du sujet : leur provenance étrangère constitue l'unique point commun. Édités à l'origine hors d'Argentine dans des imprimés locaux, ils en furent extraits par le comité de rédaction de $\mathrm{La}$ Vanguardia et publiés dans le journal, après avoir été traduits en espagnol - sauf quelques brochures qui seront analysées à part ci-dessous. Malheureusement, il est impossible de déterminer précisément combien furent traduits à Buenos Aires par des militants du socialisme argentin et combien furent traduits dans d'autres espaces hispanophones, car les sources ne l'indiquent presque jamais.

Le tableau 1 présente un aperçu du corpus de titres sur lequel s'appuie cette recherche. Le graphique 1, quant à lui, représente l'évolution par année de la publication des articles d'origine étrangère. Étant don-

formation en journal quotidien au milieu de la première décennie du $\mathrm{XX}^{\mathrm{e}}$ siècle, voir Juan Buonuome, "Fisonomía de un semanario socialista : La Vanguardia, 1894-1905 ", in Archivos de historia del movimiento obrero y la izquierda, $\mathrm{n}^{\circ}$ 6, 2015, pp. 11-30 et "Los socialistas argentinos ante la "prensa burguesa ": El semanario La Vanguardia y la modernización periodística en la Buenos Aires de entresiglos ", Boletín del Instituto de Historia Argentina y Americana "Dr Emilio Ravignani", $\mathrm{n}^{\circ}$ 46, 2017, pp. 147-179.

9 La liste des 311 articles est disponible ici : https://bit.ly/2XAIA3N. Nous rappelons au lecteur qui utilise cette source l'obligation de la mentionner. 
né que, comme nous l'avons déjà mentionné, le nombre de numéros publiés chaque année ne fut pas toujours le même ${ }^{10}$, il nous a semblé pertinent, comme le montre le graphique 2 , d'évaluer la moyenne de textes d'origine étrangère par numéro publié, même si cette donnée ne modifie quasiment pas l'évolution générale.

Tableau 1. Nombre d'articles d'origine étrangère, publiés par La Vanguardia, 1894-1905.

\begin{tabular}{|l|l|l|l|}
\hline Année & $\begin{array}{l}\text { Numéros pu- } \\
\text { bliés }\end{array}$ & $\begin{array}{l}\text { Nombre de } \\
\text { parutions } \\
\text { étrangères iden- } \\
\text { tifiées }\end{array}$ & \\
\hline 1894 & 39 & 38 & $0,97 \%$ \\
\hline 1895 & 52 & 19 & $0,37 \%$ \\
\hline 1896 & 52 & 42 & $0,81 \%$ \\
\hline 1897 & 60 & 67 & $1,12 \%$ \\
\hline 1898 & 54 & 32 & $0,59 \%$ \\
\hline 1899 & 39 & 18 & $0,46 \%$ \\
\hline 1900 & 51 & 24 & $0,47 \%$ \\
\hline 1901 & 52 & 24 & $0,46 \%$ \\
\hline 1902 & 51 & 15 & $0,29 \%$ \\
\hline 1903 & 50 & 10 & $0,20 \%$ \\
\hline 1904 & 53 & 17 & $0,32 \%$ \\
\hline 1905 & 33 & 5 & $0,15 \%$ \\
\hline TOTAL & 586 & 311 & $0,53 \%$ \\
\hline
\end{tabular}

Source : La Vanguardia

10 En 1894, le journal fut publié pour la première fois en avril ; en 1905, l'analyse s'interrompt à la fin du mois d'août, lorsqu'il change de format et de style pour devenir un quotidien ; en 1899, il manque trois mois de publications dans les archives. 
Graphique 1. Nombre de publications étrangères par année

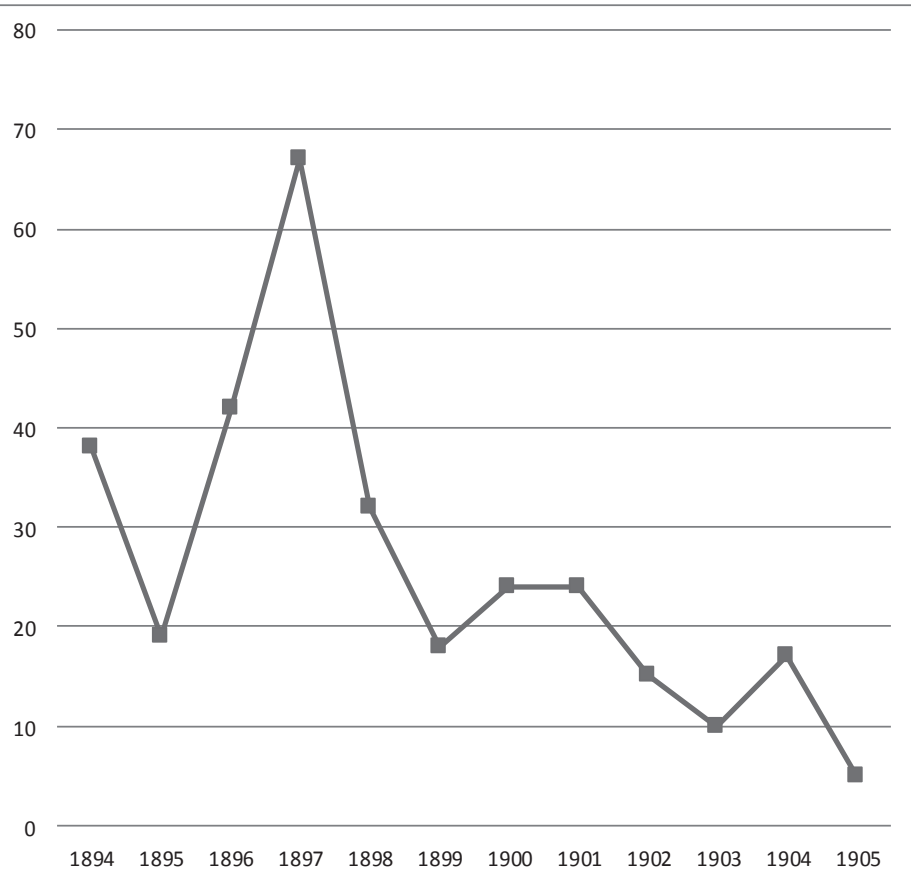

Graphique 2. Moyenne des publications étrangères par numéro.

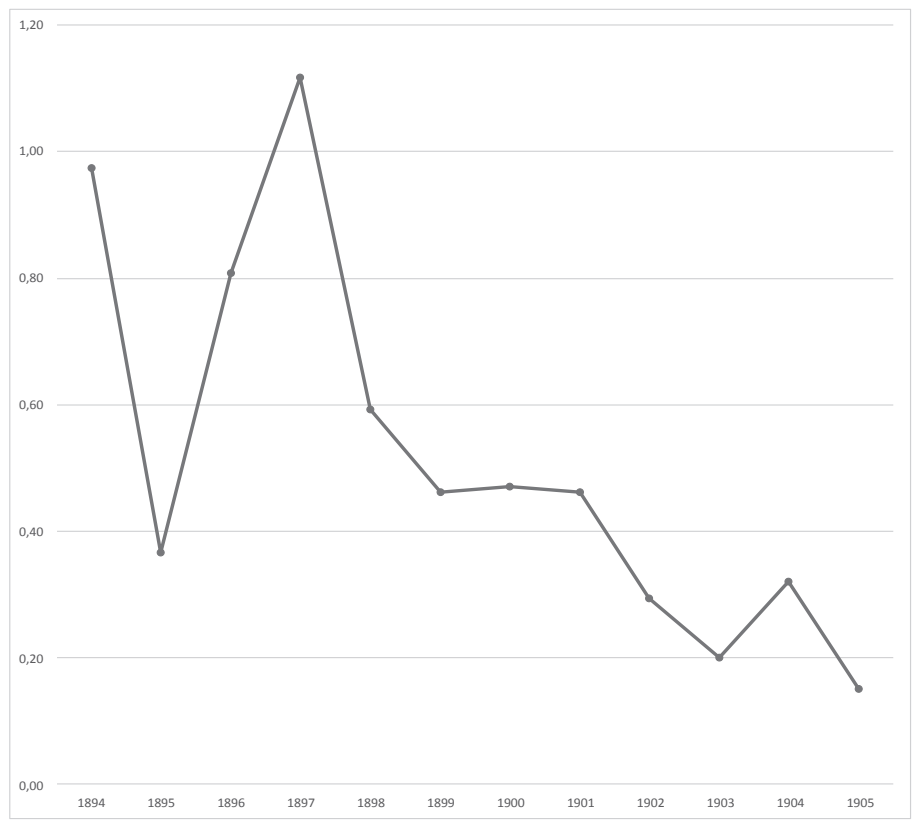


On peut tirer quelques conclusions de ce premier état des lieux purement quantitatif du nombre d'articles étrangers publiés dans le journal socialiste argentin, au stade précoce de son histoire. Tout d'abord, il est évident que, bien que nombreux, les articles produits à l'étranger ne représentaient qu'une faible proportion du nombre total d'articles publiés dans La Vanguardia : en moyenne 0,53 article par numéro sur toute la période étudiée, dans un périodique qui contenait toujours au moins une douzaine d'articles. Si l'influence des idées étrangères - généralement jugées, par ceux qui identifiaient le socialisme à une «fleur exotique " inadaptée aux intérêts des classes populaires locales - ne peut être réduite au nombre d'articles traduits et publiés par la presse du parti, on peut cependant questionner l'imputation faite à ce journal socialiste de ne diffuser que du matériel de propagande étranger et ainsi constater que cette accusation ne résiste pas aux preuves empiriques présentées ici.

Outre cette clarification concernant la proportion d'articles d'origine étrangère par rapport aux articles écrits localement, les données permettent également de tirer certaines conclusions quant à leur évolution au fil des ans. En ce sens, on remarque que la présence d'articles d'origine étrangère dans la presse du parti socialiste argentin diminua progressivement mais régulièrement vers la fin du XIXe siècle et au début de la décennie suivante. La tendance générale, en effet, rend compte d'une période initiale au cours de laquelle la publication de documents d'origine étrangère fut plus importante et d'une étape ultérieure au cours de laquelle le parti se consolida et le poids des traductions et publications européennes diminua.

Comment interpréter cette évolution? Deux raisons expliquent ce mouvement. Tout d'abord, au cours des premières années, la publication de textes écrits par des auteurs "confirmés " évoluant dans des contextes politiques consolidés, répondait au besoin de s'appuyer sur une tradition plus solide et de renforcer ainsi la crédibilité des petits groupes minoritaires du socialisme local. Deuxièmement, il semble y avoir un rapport inversé entre d'une part la quantité de propagande traduite ou reproduite, extraite de la presse étrangère, et de l'autre la participation du PS aux mouvements de lutte de la classe ouvrière locale. En effet, si l'on compare l'évolution des traductions à d'autres graphiques quantitatifs représentant les performances électorales du PS 
au cours de cette période, on peut observer que plus le parti était faible, plus le nombre d'articles de propagande d'origine étrangère - extraits/ traduits/reproduits - était élevé. Et lorsque le parti se développa, ce type de publications perdit de l'espace et les articles écrits par les militants locaux gagnèrent du terrain. C'est ce que révèle la comparaison entre les graphiques $1 / 2$ et le graphique 3, qui montre l'évolution des articles locaux du parti au cours de cette même période. Cela expliquerait aussi la diminution importante des articles d'origine étrangère en 1895, année au cours de laquelle les militants socialistes locaux étaient très mobilisés dans divers combats, l'organisation de grèves requérant toute l'attention du journal.

\section{Graphique 3. Articles locaux du Parti socialiste}

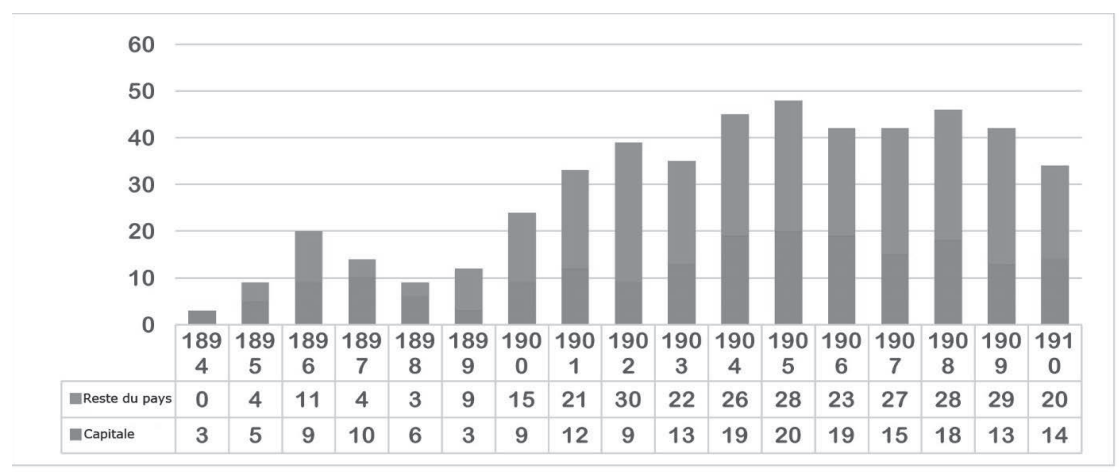

Les auteurs des documents publiés et leurs pays d'origine

Qui étaient les auteurs des documents étrangers publiés par La Vanguardia à cette époque ? De quelles publications ces documents étaientils extraits? Les réponses à ces questions apportent quelques éléments importants concernant non seulement la compréhension des réseaux de diffusion du socialisme européen à cette époque, mais aussi les préférences politiques des éditeurs de la presse socialiste argentine.

Il convient ici de préciser la méthode. Comme mentionné ci-dessus, parmi les articles de la presse étrangère traduits et reproduits qui constituent le corpus de cet article, un grand nombre n'indique ni les publications dont ils sont extraits, ni les auteurs d'origine. Ci-dessous, nous nous concentrerons donc sur un plus petit nombre d'articles : sur 
l'échantillon initial de 311 textes, seuls 101 contiennent des références au journal d'origine et 244 indiquent qui en est l'auteur : les tableaux et graphiques ci-dessous sont basés sur ceux-ci. Quoiqu'il en soit, il s'agit d'une quantité relativement importante - répartie uniformément sur les différentes années de l'échantillon - qui permet de tirer un certain nombre de conclusions pertinentes.

Dans quels pays les articles reproduits par La Vanguardia étaient-ils publiés à l'origine ? Comme le montre le graphique 4, $40 \%$ des articles indiquant leur source d'origine proviennent de journaux italiens, $23 \%$ de publications espagnoles et $13 \%$ de magazines et journaux français. En d'autres termes, près des deux tiers des documents reproduits provenaient d'Italie et d'Espagne, confirmant le rôle de ces pays dans la circulation de la propagande écrite, souvent liée à la migration des travailleuses et travailleurs. Si l'on y ajoute les sources françaises, on constate que le socialisme « latin» fournissait plus des trois quarts du total des textes réédités par la presse socialiste argentine de l'époque. Notons la présence relativement faible de la presse allemande, en dépit du fait qu’à l'époque la social-démocratie de ce pays jouait un rôle de premier plan au sein de la social-démocratie internationale.

Graphique 4. Pays d'origine des articles étrangers.

Quantité d'articles

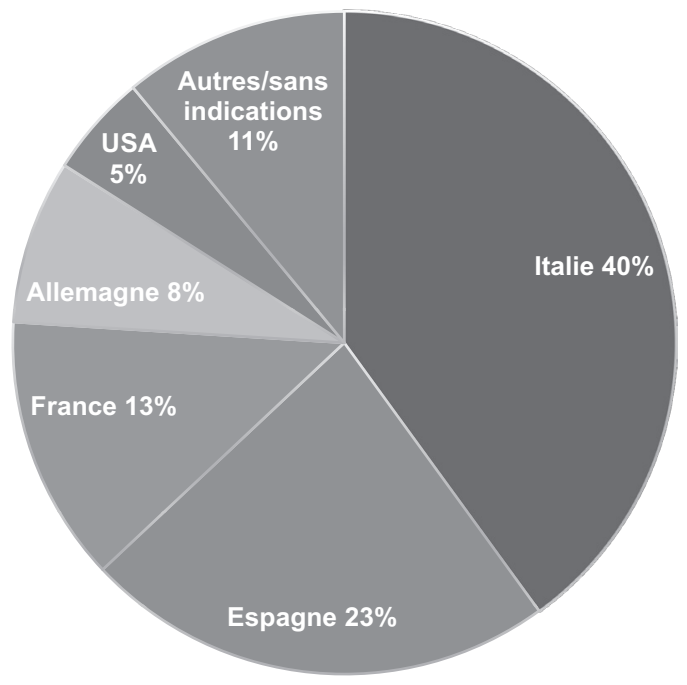


Il convient désormais d'examiner de plus près la provenance de ces articles. Le tableau suivant met en évidence, sur un total de 101 textes analysés ici, tous les périodiques étrangers dont au moins trois articles furent publiés par La Vanguardia. Comme on peut le constater, deux journaux alimentaient un tiers du total des articles : Critica Sociale de Milan, fournissait $18 \%$ des articles, et El Socialista de Madrid, $14 \%$. Et, en ajoutant les $8 \%$ issus de la Lotta di Classe, également publié à Milan, et les $6 \%$ de La lucha de clases de Bilbao, l'ensemble de ces journaux représentaient la source principale de presque la moitié du total des articles étrangers - dont l'origine était mentionnée - publiés par $\mathrm{La}$ Vanguardia pendant cette période.

Tableau 2. Journaux sources des articles d'origine étrangère

publiés dans La Vanguardia

\begin{tabular}{|l|l|}
\hline Périodique & Articles \\
\hline Critica Sociale (Milan) & 19 \\
\hline El Socialista (Madrid) & 14 \\
\hline Lotta di Classe (Milan) & 8 \\
\hline La lucha de clases (Bilbao) & 6 \\
\hline Die Neue Zeit (Stuttgart) & 5 \\
\hline Il Grido del Popolo (Turin) & 3 \\
\hline Il Socialismo (Roma) & 3 \\
\hline La Petite République (Paris) & 3 \\
\hline L'Era Nuova (Gênes) & 3 \\
\hline The People (New York) & 3 \\
\hline Autre & 34 \\
\hline
\end{tabular}

Rien n'indique cependant que les textes que l'on considère comme les originaux furent effectivement écrits et publiés pour la première fois dans les périodiques des pays mentionnés : souvent, ils étaient euxmêmes le fruit d'une ou plusieurs traductions successives et avaient déjà circulé à travers différents pays. Si l'on passe de l'analyse des journaux à l'examen des auteurs, on obtient - comme déjà mentionné - un total de 244 articles signés. Quels étaient donc les auteurs des articles que publiait La Vanguardia à cette époque?

Pour première remarque, on observe une écrasante majorité d'auteurs masculins. Seulement huit articles (3,2\% de l'échantillon) furent écrits 
par des femmes : les Françaises Lucile Desmoulins et Marie Bonnevial, la Belge Lalla Kufferath et l'Italienne Emilia Marabini (auteure de cinq articles). Ceux-ci étaient tous consacrés, d'une manière ou d'une autre, à des questions relevant de l'organisation des travailleuses et à la question dite "des femmes». Le tableau 3 montre quels furent les auteurs les plus prisés par La Vanguardia. La figure 5 organise ces informations en fonction de leurs pays d'origine.

Tableau 3. Auteurs étrangers les plus publiés par La Vanguardia

\begin{tabular}{|l|l|}
\hline Auteur & Articles \\
\hline De Amicis, Edmondo & 19 \\
\hline Vandervelde, Émile & 13 \\
\hline Turati, Filippo & 11 \\
\hline Morato, Juan José & 9 \\
\hline Prampolini, Camillo & 8 \\
\hline Spencer, Herbert & 8 \\
\hline Lafargue, Paul & 7 \\
\hline Letourneau, Charles & 7 \\
\hline Zola, Émile & 7 \\
\hline Engels, Friedrich & 5 \\
\hline Ferri, Enrico & 5 \\
\hline Iglesias, Pablo & 5 \\
\hline Kautsky, Karl & 5 \\
\hline Loria, Achille & 5 \\
\hline Marabini, Emilia & 5 \\
\hline Bebel, August & 4 \\
\hline Bernstein, Eduard & 4 \\
\hline Guesde, Jules & 4 \\
\hline Jaurès, Jean & 4 \\
\hline Bissolati, Leonida & 3 \\
\hline Deville, Gabriel & 3 \\
\hline Liebknecht, Wilhelm & 3 \\
\hline Marx, Karl & 3 \\
\hline Plekhanov, Gueorgui & 3 \\
\hline Zerboglio, Adolfo & 3 \\
\hline & \\
\hline
\end{tabular}


Graphique 5. Pays d'origine des auteurs publiés dans La Vanguardia

Pays d'origine des auteurs.

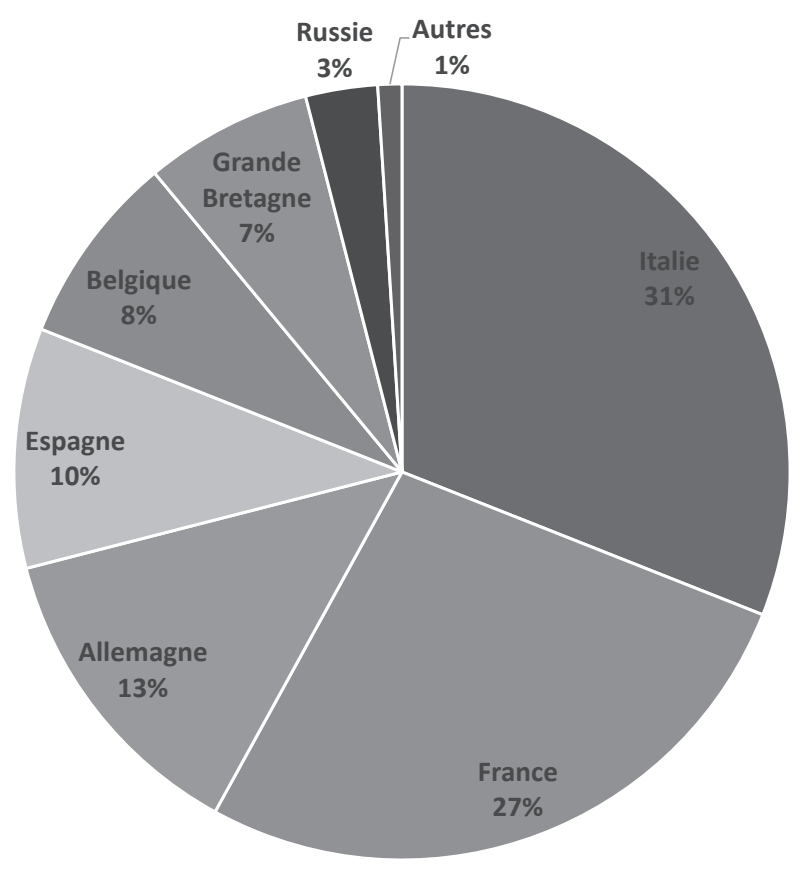

On peut tirer de nombreuses conclusions et interprétations de ces données. La première remarque consiste à nuancer la forte prédominance italienne et espagnole. $40 \%$ des articles dont la source était mentionnée provenaient de journaux italiens et $23 \%$ de publications espagnoles, mais, comme le montre le graphique 5, les auteurs italiens ne représentent que $31 \%$ et les espagnols $10 \%$. Cela s'explique par le fait que la presse italienne et espagnole était en réalité un support que les auteurs d'autres pays utilisaient pour diffuser leurs textes. À Buenos Aires, ces articles étaient soit traduits dans la langue locale, soit disponible directement en italien, langue très répandue à l'époque. La présence d'auteurs allemands était encore relativement faible, $15 \% \mathrm{du}$ total, mais elle était plus significative que la proportion d'articles tirés directement de journaux allemands, qui, comme nous l'avons vu plus haut, était de $8 \%$. Comme le souligne Tarcus, "même si l'équipe de rédacteurs de La Vanguardia disposait de traducteurs allemands (...), quand il s'agissait de passer de la Verein Vorwärts au parti socialiste, la 
politique de traduction du journal favorisait le socialisme latin au socialisme allemand ${ }^{11}$ ".

Comme on peut le voir, l'auteur le plus souvent reproduit était l'italien Edmondo de Amicis, romancier très populaire à l'époque et militant du socialisme italien. Sa présence récurrente dans les pages de La Vanguardia montre l'importance pour les éditeurs et les lecteurs de la presse socialiste de l'époque d'une certaine forme de littérature et permet de saisir combien les textes étrangers reproduits en Argentine n'étaient pas nécessairement des textes de propagande et encore moins ce qui allait devenir des "classiques " du marxisme, textes canoniques que par la suite toutes les bibliothèques militantes allaient se procurer. On peut dire la même chose d'Herbert Spencer, dont l'omniprésence révèle la forte influence idéologique parmi les dirigeants socialistes de cette période - comme le montre la bibliographie.

On retiendra aussi l'importance du belge Émile Vandervelde et de l'italien Filippo Turati dans cette phase de formation du socialisme argentin : systématiquement, la rédaction du journal argentin les considérait comme des porte-paroles légitimes et s'appuyait sur leurs textes pour propager des projets socialistes capables de répondre à la multitude de questions sociales et politiques. Comme indiqué ci-dessus, la présence des textes de Vandervelde ne correspondait nécessairement pas à une forte référence aux journaux belges, ce qui montre que cet auteur était lu par l'intermédiaire de la presse d'Europe du Sud, notamment italienne. De même, à plus petite échelle, on peut dire la même chose de deux autres propagandistes importants du socialisme italien et espagnol, respectivement Camillo Prampolini et Juan José Morato.

On note que Karl Marx, et dans une moindre mesure Friedrich Engels, sont absents de la liste. En effet, même s'ils furent traduits, comme nous le verrons plus bas, sous forme de brochures et de livres, la propagande déployée par les socialistes argentins de l'époque, s'appuyait avant tout sur une multitude d'auteurs et de dirigeants actifs dans la socialdémocratie de leur temps - les italiens Enrico Ferri, Emilia Marabini ou Leonida Bissolati, les allemands August Bebel, Eduard Bernstein ou Karl Kautsky, l'espagnol Pablo Iglesias, les français Jules Guesde, Jean Jaurès ou Gabriel Deville - plutôt que sur des textes classiques du fondateur du socialisme scientifique et de son principal collaborateur.

11 Horacio Tarcus, Marx en la Argentina..., op. cit., p. 327. 


\section{Vente de brochures}

Outre les données issues des articles et des textes reproduits dans les pages de La Vanguardia, le journal fournit également d'autres informations précieuses : la recension des brochures et petits livres mis en vente dans les locaux socialistes. Ce type de brochures était une composante fondamentale de l'activité politique et propagandiste des socialistes de l'époque. Elles étaient habituellement de petite taille et très peu coûteuses : plus encore que le journal - généralement plus axé sur les questions d'actualité - ces documents étaient considérés comme des éléments clés dans la formation politique des militants et des sympathisants. Horacio Tarcus a exploré d'ailleurs l'univers de ces publications, leur rôle dans la configuration des partis sociaux-démocrates de cette période et la relation complexe qui s'établissaient entre les initiatives éditoriales locales et celles des organisations politiques elles-mêmes ${ }^{12}$.

L'analyse des 586 numéros de La Vanguardia qui constituent le corpus de sources de ce travail, a permis d'identifier un total de 142 livres et brochures, référencés dans les pages du journal. Cependant, cette liste ne représente pas nécessairement toutes les brochures effectivement à la disposition des militants socialistes de l'époque. Il est en effet possible que d'autres brochures, non mentionnées dans le journal, aient été mises en vente, de même qu'il se peut également que certaines brochures référencées dans La Vanguardia n'aient en réalité jamais été disponibles. D'autre part, les brochures à vendre ne représentaient qu'une partie du matériel de propagande de ce type, à disposition des militants socialistes et de leurs sympathisants. En effet, les bibliothèques locales prêtaient de nombreux autres livres et brochures destinés à l'information, documentation qu'en l'absence de source, il est impossible d'analyser ici. Nous n'étudions pas non plus les éditeurs et organisations qui publiaient et traduisaient chacune de ces brochures, question essentielle mais qui a été déjà soigneusement examinée par Tarcus ${ }^{13}$. L'intention de cette partie est de valoriser une série de données générales sur les titres référencés dans le périodique argentin servant à compléter l'analyse menée jusqu'ici sur la reproduction d'articles étrangers dans La Vanguardia ${ }^{14}$.

12 Ibid., pp. 329-342.

13 Ibidem.

14 La liste des brochures à vendre est disponible ici : http://bit.ly/2XxXH9k. 
Quels titres et quels auteurs étaient favorisés à cette époque ? Quelles informations peut-on tirer des 142 brochures annoncées à la vente dans le journal ? La liste des titres, comme on peut le constater, est très hétérogène et comprend à la fois des extraits d'ouvrages classiques et des petites brochures de propagande, citant certains des textes fondamentaux de Karl Marx et de Charles Darwin, l'histoire de la Révolution française de Michelet et plusieurs œuvres de Haeckel. 16 \% du nombre total de brochures ne concerne pas ce travail, car il s'agit de textes écrits par des militants argentins : sept sont signés par Juan B. Justo, sept par Adrián Patroni, quatre par José Ingenieros et le reste par Alfredo Palacios, Augusto Bunge, Gabriela Laperrière, Manuel Ugarte et Carlos Malagarriga. La proportion de femmes auteures est encore plus faible que pour les articles : seulement 4 brochures (moins de $3 \%$ du total) furent écrites par des femmes : Ida van Etten, Pilar Taboada, Leda Rafanelli et Gabriela Laperrière.

Le tableau suivant montre quels étaient les auteurs étrangers les plus populaires figurant dans la liste des 119 titres, tout en excluant les brochures produites en Argentine. Nous nous intéressons à tous les auteurs dont le nom est mentionné au moins deux fois dans la liste générale.

Tableau 4. Auteurs étrangers d'au moins deux brochures $r$ éférencées dans La Vanguardia.

\begin{tabular}{|l|c|}
\hline Auteur & Nombre de titres \\
\hline Marx, Karl & 8 \\
\hline Turati, Filippo & 7 \\
\hline De Amicis, Edmondo & 5 \\
\hline Guesde, Jules & 4 \\
\hline Lafargue, Paul & 4 \\
\hline Vandervelde, Émile & 4 \\
\hline Darwin Charles & 3 \\
\hline Deville, Gabriel & 3 \\
\hline Haeckel, Ernst & 3 \\
\hline Jaurès, Jean & 3 \\
\hline Plekhanov, Gueorgui & 3 \\
\hline Reclus, Élisée & 3 \\
\hline
\end{tabular}




\begin{tabular}{|l|l|}
\hline Aquino, M. & 2 \\
\hline Ardigò, Roberto & 2 \\
\hline Büchner, Ludwig & 2 \\
\hline Engels, Friedrich & 2 \\
\hline Morato, Juan José & 2 \\
\hline Nordau, Max & 2 \\
\hline Tolstoi, Leon & 2 \\
\hline
\end{tabular}

Ce tableau montre des résultats proches de ceux obtenus à l'examen des articles publiés dans le journal. Cependant, on observe que les textes de Marx sont plus présents dans les livres et les brochures que dans les textes - plus courts - publiés chaque semaine dans le périodique. On retrouve aussi, comme pour les articles, la forte influence d'Edmondo de Amicis pour la culture socialiste de l'époque, de Vandervelde et de Turati.

Enfin, il résulte de la comparaison des graphiques 5 et 6 , que les auteurs étrangers des brochures référencées par La Vanguardia étaient majoritairement français, tandis que ceux qui étaient le plus reproduits dans la presse périodique, étaient principalement italiens. D'un autre côté, remarquons que 23 brochures (soit près de $20 \%$ du matériel provenant d'auteurs étrangers) furent publiées directement en italien. Elles furent très importantes dans la période 1894-1896 mais elles disparurent quasiment complètement durant les années suivantes. Cela montre que, lorsque le socialisme argentin commença à se consolider, la publication de documents en espagnol (édités en Argentine ou bien importés d'Espagne) fut privilégiée. Cela révèle également qu'à la fin du $\mathrm{XIX}^{\mathrm{e}}$ siècle, la diffusion de textes en italien dans les milieux ouvriers de Buenos Aires était monnaie courante. L'italien était cependant la seule langue étrangère des brochures proposées par La Vanguardia. 
Graphique 6 : Pays d'origine des auteurs de brochures référencées par La Vanguardia

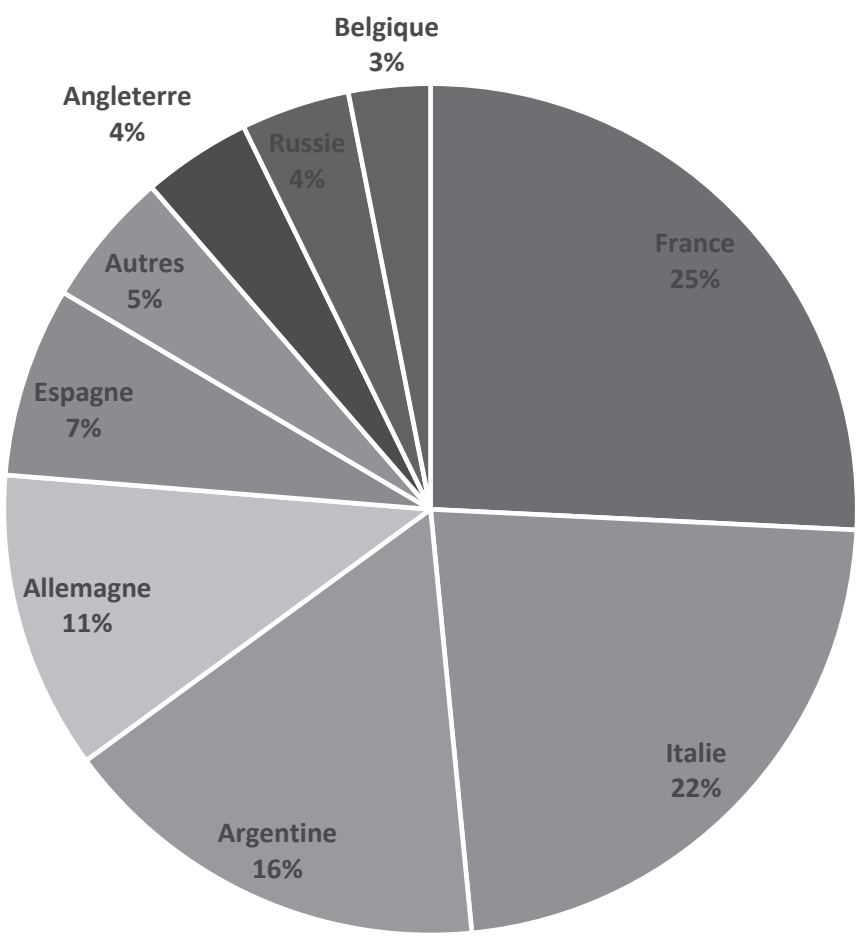

Les analyses développées dans cet article se présentent comme une contribution aux études sur la diffusion du socialisme à l'époque de la Deuxième Internationale, en général, et sur l'accueil des textes et auteurs étrangers par le parti socialiste argentin à la fin du XIX siècle et au début du XXe, en particulier. Il s'agit, bien entendu, d'un premier apport qui requiert quelques relectures et interprétations plus approfondies. Ce travail vise à donner un aperçu exhaustif des articles traduits et publiés dans La Vanguardia et des brochures vendues par le parti. Lobjectif est de proposer une série de données quantitatives, à disposition d'analyses ultérieures, concernant l'influence des textes étrangers sur le socialisme local, la nationalité des auteurs traduits et diffusés et les journaux étrangers qui constituaient pour le parti socialiste argentin, la source principale de textes propagandistes et théoriques. 
L'examen de ce corpus de sources permet de tirer un certain nombre de conclusions. Tout d'abord, même si les textes produits à l'étranger avaient une place importante dans le journal officiel du socialisme argentin, ils étaient loin d'être l'élément dominant. Comme l'avaient déjà constaté en effet les lecteurs de La Vanguardia à l'époque, la grande majorité des articles et des chroniques étaient écrites par les militants locaux. Les chiffres fournis dans cet article montrent qu'en moyenne, il y avait tout au plus un article de provenance étrangère par numéro, alors que le journal comportait quatre pages proposant chacune quatre ou cinq articles relativement longs, sans compter le grand nombre de récits courts, de chroniques, d'informations organisationnelles, d'invitations et de publicités. D'autre part, nous avons montré combien la quantité de documents traduits et republiés baissait au fil des ans, ce qui prouve la consolidation de l'organisation locale du parti. Le contrôle sur l'élaboration de ses propres textes de propagande réduisait ainsi sa dépendance aux écrits étrangers.

Comme nous l'avons vu, les informations concernant les sources à partir desquelles La Vanguardia puisait ses textes sont très riches et feront l'objet d'autres recherches à venir. Cependant, il est d'ores et déjà possible d'élaborer des propositions d'analyse. Il est indéniable que les journaux italiens et espagnol jouaient un rôle essentiel dans la propagande socialiste, puisqu'ils étaient non seulement lus par les militants argentins mais aussi diffusés dans le pays. Les données présentées dans cet article - révélant qu'il y avait beaucoup plus de sources tirées de journaux espagnols et italiens que d'auteurs provenant de ces pays montrent que les organes de presse socialistes de ces deux pays étaient des vecteurs essentiels de la diffusion des idées et des stratégies, souvent élaborées à l'origine par des dirigeants socialistes d'autres pays européens. Par exemple, Critica Sociale de Milan, et El Socialista de Madrid, étaient particulièrement bien représentés. Il reste donc à analyser plus spécifiquement, ce qui fera l'objet d'analyses ultérieures, les relations de ces deux publications capitales avec le socialisme « latin " de l'époque.

L'analyse des noms des auteurs étrangers qu'à l'époque La Vanguardia sélectionnait, référençait, et parfois traduisait, publiait et diffusait, révèle que les socialistes argentins s'appuyaient principalement sur les textes de dirigeants de partis sociaux-démocrates européens de l'époque, et dans une moindre mesure sur les œuvres plus anciennes de Marx et 
Engels. Ils se référaient enfin à une myriade d'auteurs populaires du moment mais qui n'étaient certainement pas marxistes : de Herbert Spencer à Charles Darwin, en passant par Ernst Haeckel et Léon Tolstoï. La prédominance des journaux italiens et espagnols ne signifiait pas que les auteurs des textes publiés étaient nécessairement originaires de ces pays : au contraire, il y avait en effet davantage de Français et d'Allemands. Cette remarque n'altère cependant en rien les conclusions générales de cette recherche : les pays du Sud de l'Europe jouèrent en effet un rôle considérable dans l'histoire du socialisme argentin.

Les analyses et conclusions présentées ici ne prétendent en aucun cas clore le débat ou apporter des réponses définitives. L'objectif plus modeste de cet article est de présenter des données ayant été organisées de manière systématique à partir d'une étude exhaustive de La Vanguardia. Il contribue ainsi à illustrer le kaléidoscope complexe d'influences intellectuelles qui, à travers la circulation des textes et des personnes venant d'Europe, façonna le socialisme du Rio de la Plata à la fin du XIX et début du XX $\mathrm{XX}^{\mathrm{e}}$ siècle. C'est en dialogue avec ces influences que les dirigeants et les militants du socialisme argentin forgèrent, à leur tour, les idéaux du socialisme de la fin du XIX et du début du $\mathrm{XX}^{\mathrm{e}}$ siècle.

Lucas POY (Instituto de historia argentina y americana

"Dr Emilio Ravignani " UBA - CONICET, Argentine) (traduit de l'espagnol par Lucie GUESNIER) 